\title{
Modelos univariados de séries temporais para previsão das temperaturas médias mensais de Erechim, RS
}

\author{
Leonardo Chechi $^{1} \&$ Fábio M. Bayer ${ }^{2}$
}

RESU M O

Este trabalho apresenta uma análise de séries temporais dos dados de temperatura mínima e temperatura máxima mensal da cidade de Erechim, RS; apresenta-se uma comparação de duas classes de modelos tradicionais de previsão, nomeadamente: modelos da classe ARIM A e modelos de alisamento exponencial. $\mathrm{Na}$ classe de modelos ARIMA foram selecionados, utilizando-se critérios de informação, modelos do tipo SARIMA, que consideram a característica sazonal da temperatura do ar; já para os modelos de al isamento exponencial utilizaram-se os modelos $\mathrm{H}$ olt-W inters aditivo, em que as constantes de al isamento são determinadas de forma a minimizar o erro quadrático médio entre valores previstos e observados; esta análise permitiu a identificação de componentes como sazonalidade e períodos atípicos. O s modelos de previsão foram comparados para diferentes horizontes de previsão, sendo que os modelos da classe ARIM A se mostraram mais acurados. 0 s modelos ajustados se mostraram adequados para traçar previsões das variáveis de temperatura do ar, mostrando-se importantes ferramentas para a climatologia agrícola.

Palavras-chave: ARIMA, alisamento exponencial, modelos de previsão, sazonalidade

\section{U nivariate time series methods for forecasting the monthly mean air temperature in Erechim, RS}

\begin{abstract}
A B STRACT
This paper presents a time series analysis of the minimum and maximum air temperature of Erechim, RS. A comparison between two traditional classes of the forecasting models, namely: ARIMA models and exponential smoothing models is also presented. In the class of ARIM A models using criteria information, SARIMA type models that consider the seasonal characteristics of air temperature were selected, whereas for exponential smoothing models Holt-W inters additive algorithm were used. Smoothing constants are determined to minimize the mean square error between observed and predicted values. This analysis allowed the identification of components such as seasonality and atypical periods. The model predictions were compared for different forecast horizons. The ARIMA class models proved to be more accurate while the adjusted models were adequate for adjusting forecasts of variables of air temperature, being important tools for agricultural climatology.
\end{abstract}

Key words: ARIMA, exponential smoothing, forecasting model, seasonality 


\section{INTRODUÇÃO}

Uma série temporal é um conjunto de observações ordenadas no tempo de qualquer fenômeno aleatório. A análise de séries temporais consiste em encontrar relações de dependência existentes temporalmente nos dados buscandose identificar o mecanismo gerador da série, com o objetivo de extrair periodicidades relevantes nas observações, descrever seu comportamento e fazer previsões (Bayer \& Souza, 2010).

A análise de séries temporais aplicada a dados climatológicos atrai interesse especial haja vista que o clima interfere diretamente em muitas áreas determinando o sucesso ou o fracasso de vários empreendimentos. Segundo Medeiros et al. (2005) na área agrícola a temperatura do ar tem influência direta em muitos efeitos fisiológicos que ocorrem nas plantas e animais, tornando essencial o desenvolvimento de estudos desses fenômenos para a tomada de decisão. Hubbard (2007) destaca, ainda, a importância das análises em climatologia agrícola sobre amplos aspectos da agricultura e pecuária. Cargnelutti Filho et al. (2008) ressaltam diversas atividades agropecuárias e ambientais que podem se beneficiar dessas análises, como o zoneamento e a análise de riscos climáticos, a orientação das atividades agropecuárias e o planejamento agrícola, a regionalização agroclimática e a potencialidade de produção das diferentes culturas, a probabilidade de atendimento hídrico das culturas, o calendário agrícola e o planejamento do plantio e colheita, o monitoramento de seca, manejo de água de irrigação, crédito e seguro agrícola.

A partir da análise de séries temporais agrícolas ou climatológicas é possível o ajuste de modelos estatísticos univariados de previsão. Tais modelos necessitam, basicamente, de um vetor de valores observados ao longo do tempo da variável climatológica de interesse. Entre os modelos tradicionais de previsão se destacam os modelos autorregressivos integrados de médias móveis (ARIMA) (Box et al., 2008) e os algoritmos de alisamento exponencial (Chatfield \& Yar, 1988). Neste trabalho essas duas classes de modelos são comparadas por meio da modelagem e previsão da temperatura do ar mensal média. Os modelos consideram características temporais que os tornam adequados para a modelagem de variáveis climáticas, tais como: (i) sazonalidade; (ii) correlação serial ao longo do tempo e (iii) acomodação de séries não estacionárias.

Modelos estatísticos têm sido utilizados de maneira satisfatória nas áreas agrícola e ambiental. Medeiros et al. (2005) estimaram as normais para temperatura do ar mensais e anuais para o nordeste brasileiro, por meio de regressão múltipla enquanto para a mesma região Cavalcanti et al. (2006) estabeleceram um modelo de estimativa da temperatura do ar através das coordenadas geográficas e das anomalias de temperaturas da superfície do mar. Silva et al. (2008) descreveram o comportamento da série de temperatura média mensal da cidade de Uberlândia, MG, através de modelos da classe ARIMA. Kärner (2009) usou modelos ARIMA para comparação da variabilidade temporal de longo alcance entre a irradiação solar total e a temperatura do ar. Soebiyanto et al. (2010) modelaram parâmetros climáticos utilizando modelos ARIMA com o objetivo de prever a transmissão de gripe em regiões frias. Bardin et al. (2010) estimaram os valores de temperatura mensais e anuais na região dos municípios que compõem o Polo Turístico do Circuito das Frutas do Estado de São Paulo, utilizando modelos de regressão. Gemitzi \& Stefanopoulos (2011) investigaram os efeitos das condições meteorológicas e das intervenções do homem sobre os aquíferos subterrâneos por meio de modelos ARIMA.

Para o estado do Rio Grande do Sul, RS, Brasil, Cargnelutti Filho et al. (2008) verificaram se a temperatura máxima média decendial e a temperatura média decendial do ar de 41 municípios podem ser estimadas em função da altitude, latitude e longitude. Ainda, Cargnelluti Filho et al. (2010) ajustaram o modelo harmônico para a estimativa da temperatura do ar média mensal em 37 locais do RS, com os dados do período de 1931 a 2000. Bayer et al. (2012) utilizam modelos da classe ARIMA para modelar e prever as vazões da bacia hidrográfica do rio Potiribu localizada na região noroeste do Estado; este último evidencia outra grande importância dos modelos estocásticos como os modelos ARIMA, na previsão de parâmetros climáticos em longo prazo. Os resultados desses modelos podem ser usados como insumo (input) em complexos modelos hidrológicos determinísticos para simulação de chuva e vazão. Ainda no que tange aos aspectos hidrológicos, a modelagem e a previsão da disponibilidade hídrica também são importantes para outros usos, como irrigação, abastecimento, navegação etc. (Colischonn et al., 2005). Referências relevantes a respeito de modelagem de séries temporais hidrológicas e suas aplicações na engenharia, são apresentadas de forma detalhada em Salas et al. (1980) e Salas (1992).

Desta maneira, percebe-se a relevância do estudo de modelos estatísticos de previsão no âmbito da engenharia agrícola e ambiental e, sendo assim, o objetivo deste trabalho é fazer um estudo de modelos de séries temporais aplicados às séries de temperaturas mínimas e máximas mensais médias da cidade de Erechim no RS. Apresenta-se uma comparação entre o desempenho das previsões dos modelos de alisamento exponencial e os modelos da classe ARIMA cujo estudo é justificado também pela escassez de dados e de estudos climatológicos quantitativos na região.

\section{Material e MÉTOdos}

Os dados em análise referem-se às séries históricas de temperaturas máximas e mínimas mensais médias da cidade de Erechim, RS. Os dados foram disponibilizados pela Fundação Estadual de Pesquisa Agropecuária (FEPAGRO) e compreendem o período de janeiro de 2003 a março de 2011. As séries temporais em estudo se restringem a um período de 8 anos e três meses, pelo fato da inexistência de séries históricas mais longas na região, totalizando 99 observações. Segundo Hyndman \& Kostenko (2007) o tamanho amostral é estatisticamente suficiente uma vez que os modelos sazonais de previsão utilizados neste trabalho possuem o número de parâmetros consideravelmente menor do que esse número de observações. As séries em estudo apresentam, ainda, comportamentos sazonais bem definidos e com pouca 
variabilidade dentro dos meses do ano, sendo adequada para o uso dos modelos abordados.

A cidade de Erechim se localiza ao norte do Rio Grande do Sul, na região do Alto Uruguai, especificamente na latitude $27^{\circ}$ 38' 3" S e longitude $52^{\circ} 16^{\prime} 26^{\prime \prime} \mathrm{W}$, com área territorial de 409,06 km² (PME, 2012). De acordo com a classificação de Köppen, o clima da cidade é do tipo Cfa, o qual se caracteriza por ser um clima subtropical úmido. As maiores temperaturas são registradas nos meses de janeiro, fevereiro e dezembro e os meses que registram as menores temperaturas são junho e julho.

Para cumprir os objetivos deste trabalho realizou-se, primeiro, uma análise descritiva dos dados; nesta análise, além de medidas descritivas foram apresentados gráficos das séries temporais, da decomposição das séries em suas componentes temporais e gráficos boxplot; enfim, esta análise descritiva preliminar é importante para a caracterização das séries e identificação de sazonalidades, tendências e valores atípicos.

Após a análise descritiva foram ajustados os modelos de alisamento exponencial (Chatfield \& Yar, 1988) do tipo HoltWinters aditivo, apresentando as constantes de alisamento e também seus valores previstos graficamente. O modelo HoltWinters aditivo é definido na Eq. 1.

$$
\mathrm{y}_{\mathrm{t}}=\mathrm{N}_{\mathrm{t}}+\mathrm{T}_{\mathrm{t}}+\mathrm{S}_{\mathrm{t}}+\varepsilon_{\mathrm{t}}, \mathrm{t}=1, \ldots, \mathrm{n}
$$

em que:

$$
\begin{aligned}
& \mathrm{E}\left(\varepsilon_{\mathrm{t}}\right)=0, \operatorname{Var}\left(\varepsilon_{\mathrm{t}}\right)=\sigma^{2} \\
& \mathrm{~N}_{\mathrm{t}} \text { - componente de nível } \\
& \mathrm{T}_{\mathrm{t}} \text { - tendência } \\
& \mathrm{S}_{\mathrm{t}} \text { - componente de sazonalidade }
\end{aligned}
$$

Desta maneira, a forma aditiva do algoritmo de Holt-Winters é apresentada nas Eqs. 2, 3 e 4.

$$
\begin{gathered}
\widehat{\mathrm{N}}_{\mathrm{t}}=\alpha\left(\mathrm{y}_{\mathrm{t}}-\widehat{\mathrm{S}}_{\mathrm{t}-\mathrm{s}}\right)+(1-\alpha)\left(\widehat{\mathrm{N}}_{\mathrm{t}-1}+\widehat{\mathrm{T}}_{\mathrm{t}-1}\right), 0 \leq \alpha \leq 1 \\
\widehat{\mathrm{T}}_{\mathrm{t}}=\beta\left(\widehat{\mathrm{N}}_{\mathrm{t}}-\widehat{\mathrm{N}}_{\mathrm{t}-1}\right)+(1-\beta) \widehat{\mathrm{T}}_{\mathrm{t}-1}, 0 \leq \beta \leq 1 \\
\widehat{\mathrm{S}}_{\mathrm{t}}=\gamma\left(\mathrm{y}_{\mathrm{t}}-\widehat{\mathrm{N}}_{\mathrm{t}}\right)+(1-\gamma) \widehat{\mathrm{S}}_{\mathrm{t}-\mathrm{s}}, 0 \leq \gamma \leq 1
\end{gathered}
$$

em que:

$\mathrm{s} \quad$ - número de vezes em que a série é observada por ano ( $\mathrm{s}=12$, pois se tem observações mensais)

$\alpha, \beta$ e $\gamma$-constantes de alisamento

Logo após, trabalhou-se com os modelos da classe ARIMA, seguindo os passos da metodologia de Box e Jenkins, nomeadamente: (i) identificação do modelo; (ii) estimação dos parâmetros e (iii) diagnóstico do modelo ajustado. Esta metodologia pode ser verificada com maiores detalhes em Morettin \& Toloi (2006) e Box et al. (2008).

Para identificação dos modelos utilizou-se também, além da análise dos correlogramas, o periodograma, que representa a série no domínio da frequência; depois de selecionado o melhor modelo é feita uma análise de diagnóstico; na classe ARIMA utilizou-se o modelo ampliado SARIMA (p,d,q)(P,D,Q) considerando-se a sazonalidade da série, conforme definido na Eq. 5.

$$
\begin{aligned}
& \left(1-\phi_{1} \mathrm{~B}-\ldots-\phi_{\mathrm{p}} \mathrm{B}^{\mathrm{p}}\right) \times\left(1-\Phi_{1} \mathrm{~B}^{\mathrm{s}}-\ldots-\Phi_{\mathrm{P}} \mathrm{B}^{\mathrm{sP}}\right) \times \\
& \times(1-\mathrm{B})^{\mathrm{d}} \times\left(1-\mathrm{B}^{\mathrm{s}}\right)^{\mathrm{D}} \mathrm{Y}_{\mathrm{t}}=\left(1+\theta_{1} \mathrm{~B}+\ldots+\theta_{\mathrm{q}} \mathrm{B}^{\mathrm{q}}\right) \times \\
& \times\left(1+\Theta_{1} \mathrm{~B}^{\mathrm{s}}+\ldots+\Theta_{\mathrm{Q}} \mathrm{B}^{\mathrm{sQ}} \varepsilon_{\mathrm{t}}\right)
\end{aligned}
$$

em que:

$\mathrm{p}, \mathrm{d}, \mathrm{q}$ - ordens do modelo referentes à dinâmica ordinal

$\mathrm{P}, \mathrm{D}, \mathrm{Q}$ - ordens referentes à parte sazonal do modelo

Os parâmetros $\phi_{1}, \ldots, \phi_{\mathrm{p}}$ e $\theta_{1}, \ldots, \theta_{\mathrm{q}}$ são, respectivamente, os parâmetros autorregressivos e de médias moveis ordinais e os parâmetros $\Phi_{1}, \ldots, \Phi_{\mathrm{P}}$ e $\Theta_{1}, \ldots, \Theta_{\mathrm{Q}}$ são os parâmetros autorregressivos e de médias móveis sazonais.

Para identificação das ordens de integração utilizou-se a análise dos correlogramas, que são gráficos das funções de autocorrelação (FAC) e da autocorrelação parcial (FACP) amostrais. Se eles decaem muito lentamente para zero há, então a indicação de que a série seja não estacionária. Mas este processo de identificação visual pode tornar-se muito difícil e trabalhoso; neste contexto são utilizados os critérios de informação ou critério de seleção de modelos.

Os critérios de seleção utilizados foram o AIC (Akaike Information Criterion) proposto por Akaike (1973), o AICc proposto por Hurvich \& Tsai (1989) e o BIC (Bayesian Information Criterion) desenvolvido por Akaike (1978). Utilizando-se esses critérios de seleção estimam-se vários modelos concorrentes e se seleciona o modelo com menor valor para o critério de informação.

Depois de identificar o melhor modelo e estimar seus parâmetros via método da máxima verossimilhança, procedese à análise de diagnóstico. O teste de Ljung-Box (Ljung \& Box, 1978) é um teste útil uma vez que ele torna possível avaliar a existência de autocorrelação nos erros por meio da autocorrelação residual. Neste teste estatístico a hipótese nula é a de que os resíduos são ruídos brancos, ou seja, não autocorrelacionados. Portanto, ao não se rejeitar a hipótese nula, ou seja, quando o p-valor decorrente do teste estatístico é maior do que um nível de significância $\alpha$ usual, conclui-se que o modelo está ajustado adequadamente.

Após a seleção e ajuste dos modelos das classes ARIMA e dos modelos Holt-Winters para as séries de temperaturas mínima e máxima tem-se o objetivo de comparar a acurácia de cada modelo; com isto, pode-se concluir qual dessas classes tradicionais de modelos captam melhor a variabilidade das séries; para isso se reservam, então, as h últimas observações para o cálculo das medidas de qualidade definidas na Tabela 1 , para $h=3$ e $h=6$, em que $y_{i}$ e $\hat{y}_{i}$ são, respectivamente, os valores reais e previstos no instante i. Também se apresenta, graficamente, uma comparação entre os $h$ valores previstos e os $\mathrm{h}$ valores observados.

Tabela 1. Medidas de qualidade de previsão

\begin{tabular}{lcc}
\hline \multicolumn{1}{c}{ Medida } & Descrição & Notação \\
$\begin{array}{l}\text { Erro quadrado } \\
\text { médio }\end{array}$ & EQM & $\frac{1}{\mathrm{~h}} \sum_{\mathrm{i}=1}^{\mathrm{h}}\left(\mathrm{y}_{\mathrm{i}}-\hat{\mathrm{y}}_{\mathrm{i}}\right)^{2}$ \\
$\begin{array}{l}\text { Erro absoluto } \\
\text { percentual médio }\end{array}$ & MAPE & $\frac{1}{\mathrm{~h}} \sum_{\mathrm{i}=1}^{\mathrm{h}} \frac{\left|\mathrm{y}_{\mathrm{i}}-\hat{\mathrm{y}}_{\mathrm{i}}\right|}{\mathrm{y}_{\mathrm{i}}}$ \\
\hline
\end{tabular}


Todos os cálculos e ajustes necessários para a realização deste trabalho foram realizados no Software Livre R ( R Development Core Team, 2009). Entre outros pacotes foram utilizados os pacotes forecast e stats, que são módulos do $\mathrm{R}$ com funções especifícas para a análise de séries temporais.

\section{RESULTADOS E DISCUSSÃO}

Para melhor compreensão das variáveis estudadas a Tabela 2 apresenta algumas medidas descritivas das séries. Verificase que a temperatura mínima mensal de Erechim tem média de $13{ }^{\circ} \mathrm{C}$ e varia de 1,8 a $18{ }^{\circ} \mathrm{C}$, sendo que a maior temperatura mínima foi registrada no mês de fevereiro de 2003 e a menor em junho de 2008; já a temperatura máxima mensal de Erechim tem média de $22,95{ }^{\circ} \mathrm{C}$, variando entre 9,2 e $29,3{ }^{\circ} \mathrm{C}$. A maior temperatura máxima foi registrada no mês de fevereiro de 2010 e a menor em junho de 2008.

Tabela 2. M edidas descritivas das séries de temperatura máxima e temperatura mínima da Cidade de Erechim

\begin{tabular}{lrc}
\hline \multicolumn{1}{c}{ Medidas } & $\begin{array}{c}\text { Temperatura } \\
\text { máxima }\end{array}$ & $\begin{array}{c}\text { Temperatura } \\
\text { mínima }\end{array}$ \\
Média & 23,000 & 13,244 \\
Mediana & 23,200 & 13,800 \\
Desvio padrão & 4,315 & 3,636 \\
Máximo & 29,300 & 18,800 \\
Mínimo & 9,200 & 1,800 \\
Coeficiente de variação & $18,76 \%$ & $27,46 \%$ \\
\hline
\end{tabular}

Outra forma de analisar descritivamente as séries estudadas é por meio da análise gráfica. A Figura 1 apresenta graficamente as séries temporais de temperatura mínima (1A) e máxima (1B) podendo-se destacar que as séries apresentam sazonalidades bem definidas.

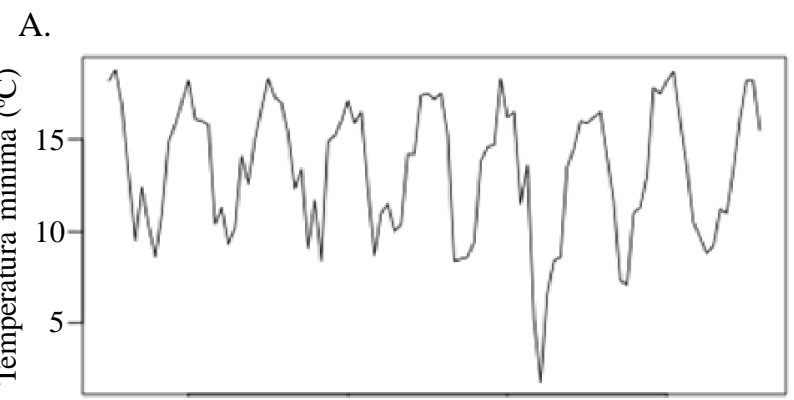

B.

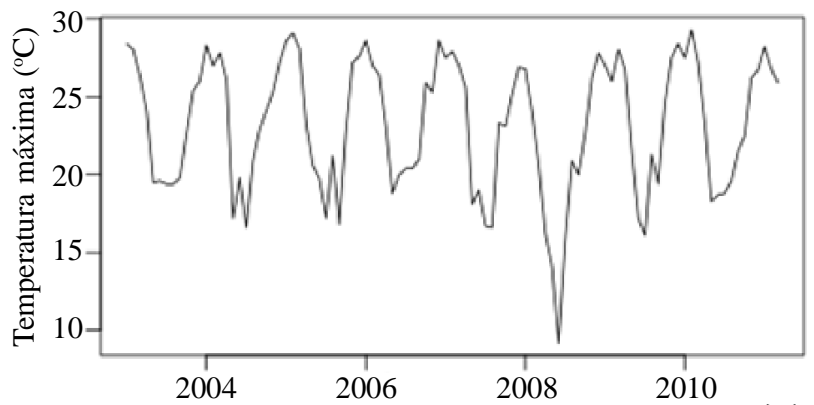

Figura 1. Séries de temperaturas mensais mínima $(A)$ e máxima (B)
A Figura 2 apresenta os gráficos de decomposição das séries de temperaturas mínima e máxima em suas componentes de tendência, sazonalidade e aleatoriedade. Observa-se que as séries tem comportamento semelhante e, corroborando com o gráfico da Figura 1, a componente sazonalidade está bem definida. Pela análise da componente tendência, verificam-se alguns períodos atípicos, como no ano de 2008, em que as temperaturas apresentaram valores mais reduzidos do que nos demais períodos.

A.

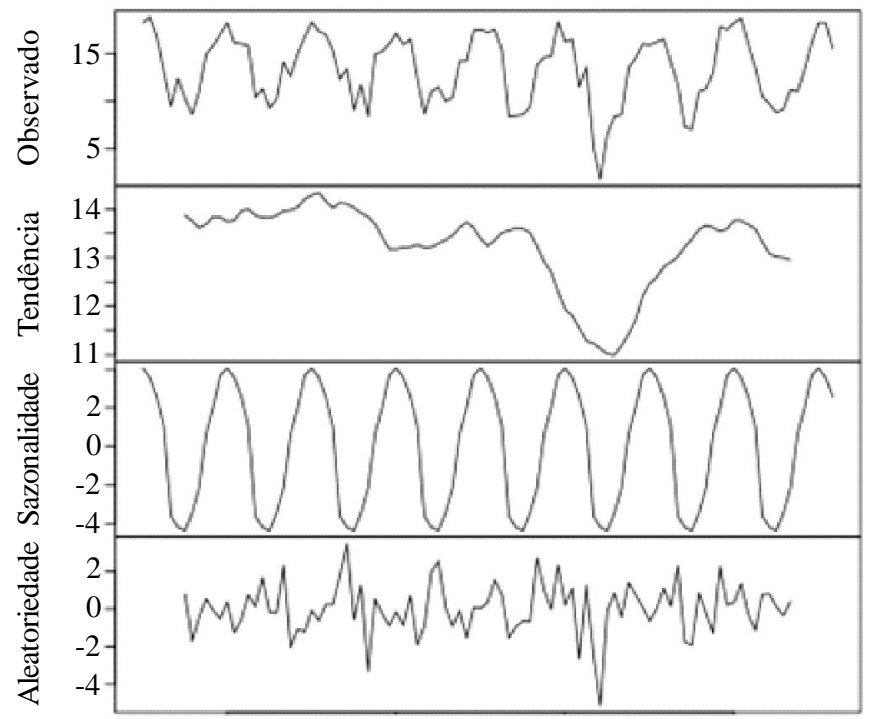

B.

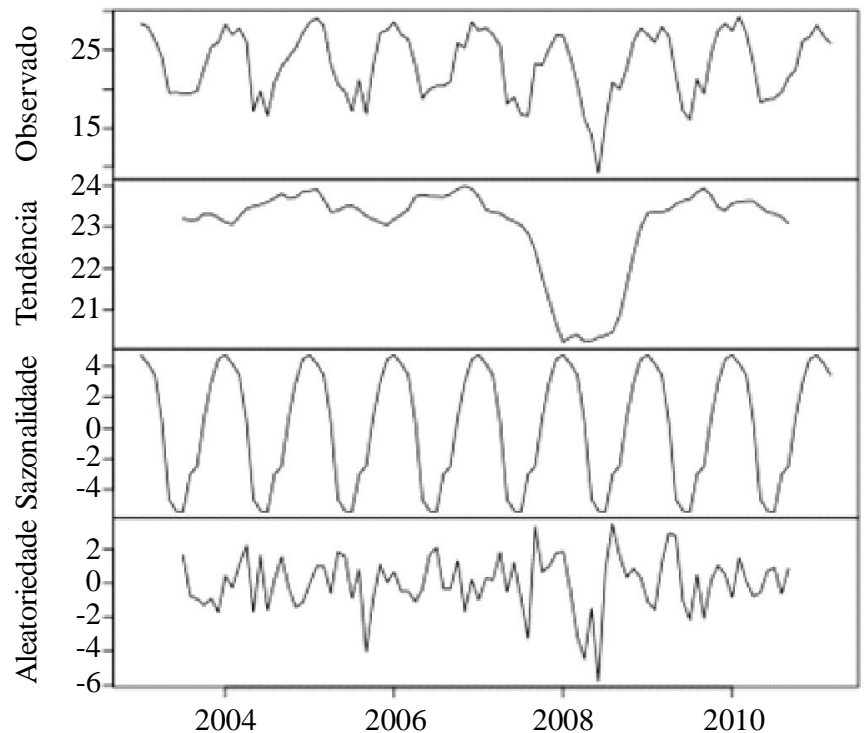

Figura 2. Gráficos de decomposição das séries de temperatura mínima (A) e temperatura máxima (B)

Outra ferramenta gráfica importante para entender as séries estudadas é o boxplot; a Figura 3 apresenta os gráficos boxplot mensais das séries de temperatura mínima e temperatura máxima. Observa-se que o mês com menor dispersão foi março e os meses com maiores dispersões foram maio e junho. Os meses de março, junho e novembro apresentaram temperaturas mínimas mensais atípicas em alguns anos, sendo que o mês de 
junho condiz com a menor temperatura mínima mensal de toda a série, igual a $1,8^{\circ} \mathrm{C}$, registrada no ano de 2008 . Para a série de temperatura máxima, observa-se comportamento semelhante ao da temperatura mínima porém é notável a existência de mais valores atípicos nos meses de fevereiro, março, abril, junho e agosto em que junho de 2008 apresenta a menor temperatura máxima da série assim como na série de temperatura mínima, com valor observado de $9,2{ }^{\circ} \mathrm{C}$; outro fator relevante a ser destacado é que os meses de janeiro, fevereiro e março apresentam menor amplitude nos dados, evidenciando a pouca variabilidade de temperatura nesses meses.

A.

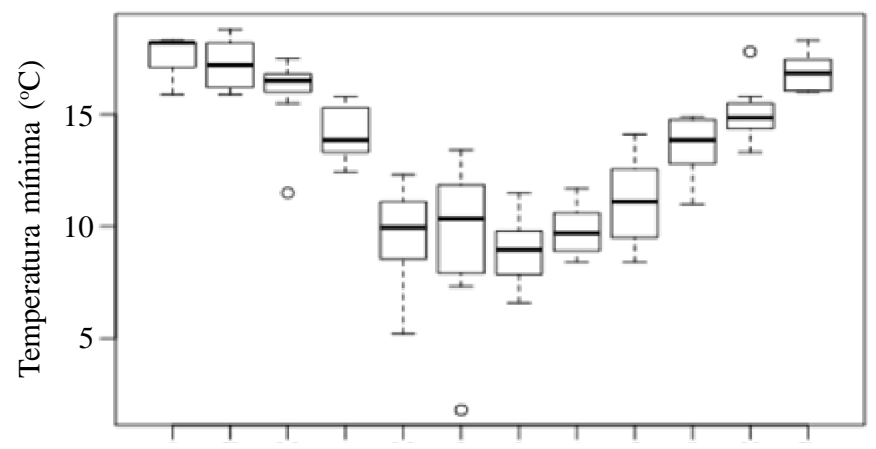

B.

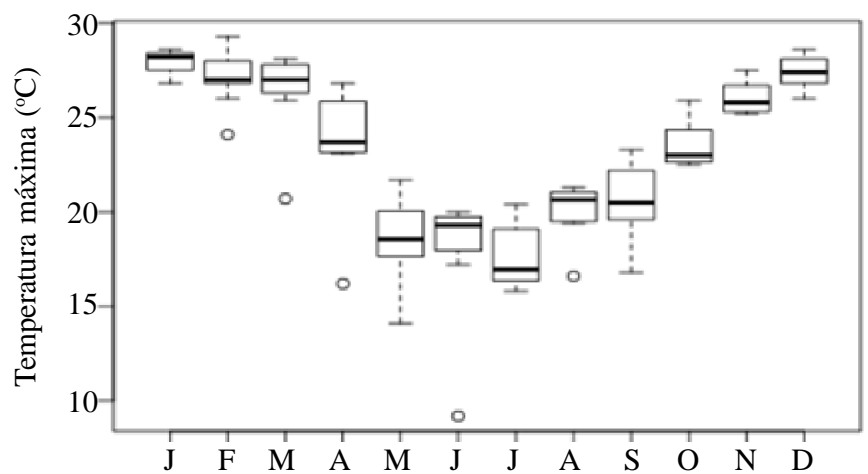

Figura 3. Boxplot mensal das séries de temperatura mínima $(A)$ e máxima $(B)$

\section{Ajuste do modelo de alisamento exponencial}

As constantes de alisamento dos modelos de alisamento exponencial, do tipo Holt-Winters aditivo, para as séries de temperaturas, são apresentadas na Tabela 3; essas constantes de alisamento minimizam o erro quadrático médio entre os valores reais e os valores previstos pelo modelo, dentro do intervalo observado. Os modelos apresentados na Tabela 3 são comparados qualitativamente na Figura 4 comparação esta que se baseia na proximidade entre a série real dos dados e a série prevista pelo modelo Holt-Winters e ainda evidencia o bom ajuste deste modelo.

\section{Ajuste do modelo da classe ARIMA}

Para a identificação dos modelos da classe ARIMA analisamse, primeiro, os correlogramas e os periodogramas das séries. Os correlogramas apresentam as correlações seriais no domínio do tempo enquanto o periodograma apresenta as características da série no domínio da frequência; este últimoé uma importante
Tabela 3. Constantes de alisamento do modelo HoltW inters para as séries de temperatura mínima e máxima

\begin{tabular}{cccc}
\hline Modelos Holt-Winters & \multicolumn{3}{c}{ Constantes de alisamento } \\
\cline { 2 - 4 } $\mathbf{H = 1 2}$ & $\boldsymbol{\alpha}$ & $\boldsymbol{\beta}$ & $\boldsymbol{\gamma}$ \\
Temperatura mínima & 0,2399 & 0,0001 & 0,0035 \\
Temperatura máxima & 0,4490 & 0,0001 & 0,0001 \\
\hline
\end{tabular}

A.

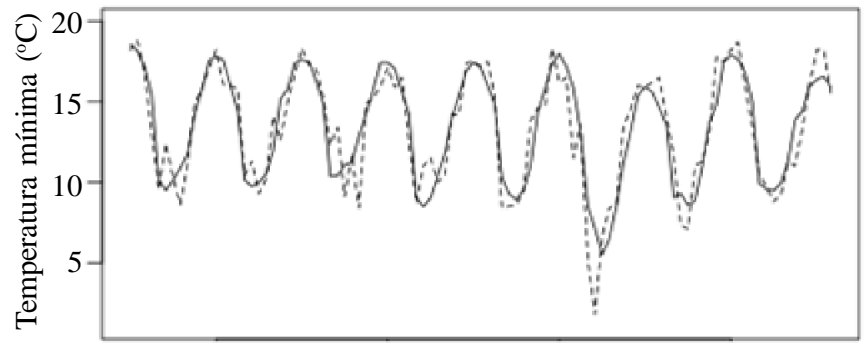

B.

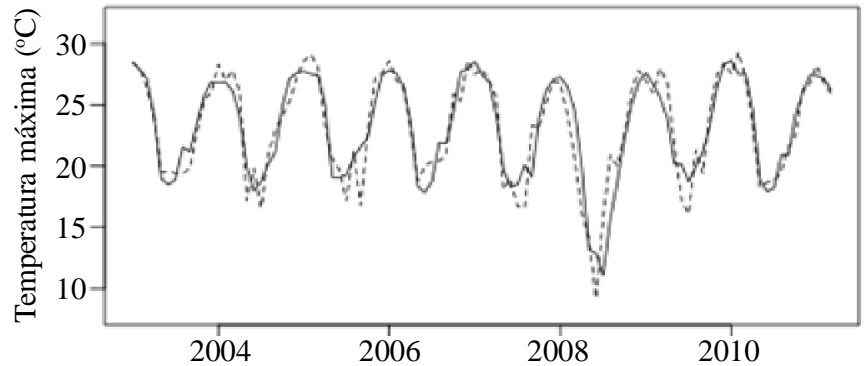

Figura 4. Valores observados e previstos para temperaturas mínima (A) e máxima (B) pelo modelo HoltW inters

ferramenta para identificar periodicidades nos dados. Com base em suas frequências estimadas é possível verificar sazonalidades e ciclos na série. A Figura 5 apresenta os correlogramas das séries de temperaturas mínima (Figura 5A) e máxima (Figura 5B) em nível, com uma diferença de ordem 1 e uma diferença de ordem 12.

Os correlogramas das duas séries apresentam comportamentos semelhantes; as FAC e FACP amostrais da série em nível apresentam maior correlação quando comparadas às FAC e FACP amostrais da série com uma diferença de ordem 1 indicando não ser necessário a aplicação desta diferença. $\mathrm{O}$ pico de correlação significativa na defasagem sazonal (1.0) que pode ser observada nas FAC e FACP amostrais com uma diferença de ordem 12, revela a presença de sazonalidade.

Analisando os periodogramas das séries de temperaturas mínima (Figura 6A) e máxima (Figura 6B), apresentados na Figura 6 observa-se, para as duas séries, que o oitavo elemento espectral é o que obtém maior valor; como há 99 observações este harmônico corresponde à periodicidade aproximada de 99/8=12,375 meses; esta periodicidade pode ser arredondada para 12 meses pois, com 99 observações, não se tem um múltiplo de 12. Desse modo, tem-se uma série com sazonalidade anual corroborando com os resultados já apresentados pelos correlogramas. A presença de sazonalidade na série indica que um modelo do tipo SARIMA deve ser considerado.

Após a determinação da classe mais ampla se estimam diversos modelos e se selecionam, via critérios de informação, 
A.

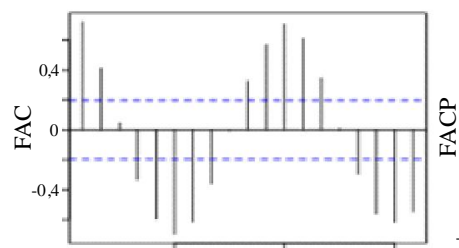

(a) FAC amostral
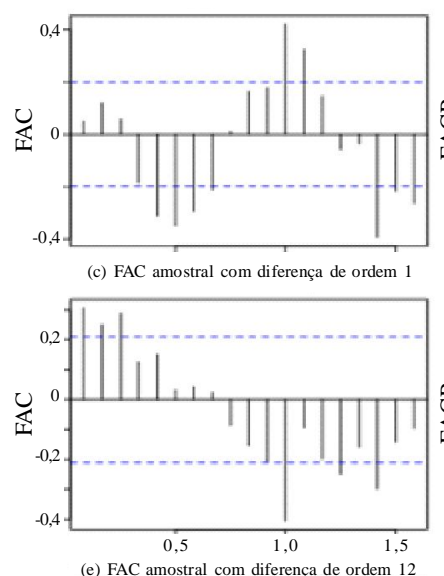

(e) FAC amostral com diferença de ordem 12

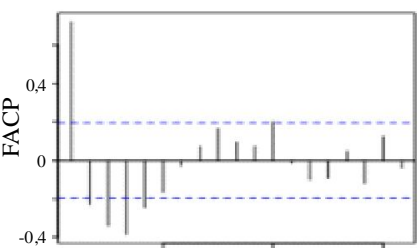

(b) FACP amostral

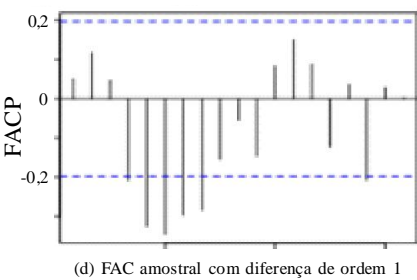

(d) FAC amostral com diferença de ordem 1

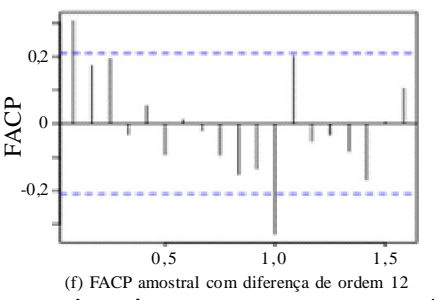

A.

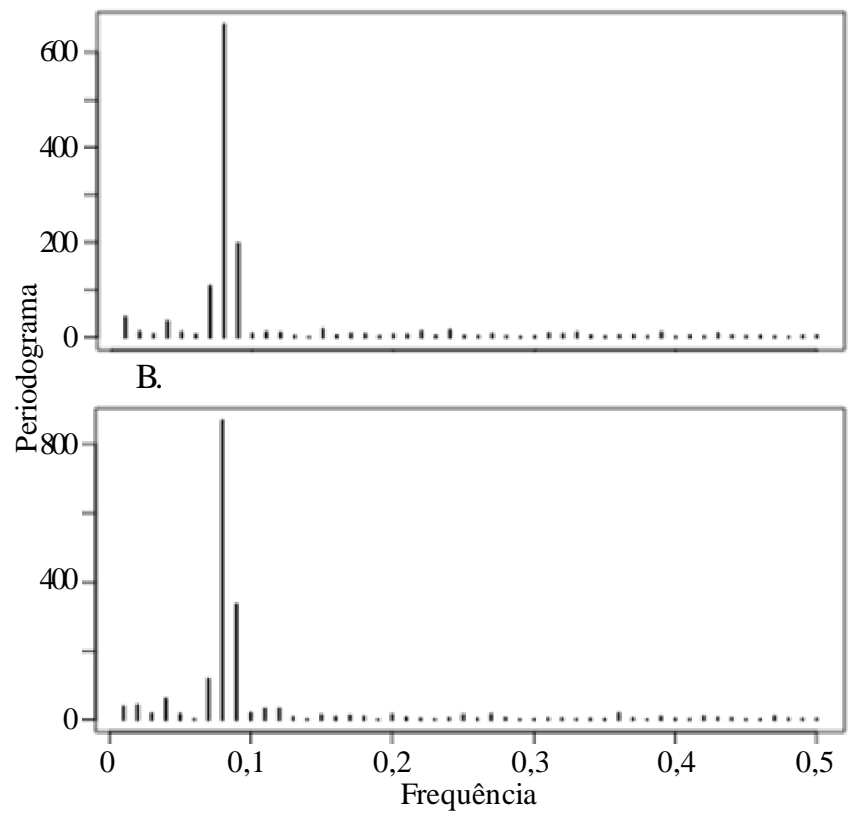

Figura 6. Periodogramas das séries de temperatura mínima (A) e temperatura máxima (B)

alguns modelos concorrentes. A Tabela 4 apresenta os melhores modelos estimados para a previsão das séries de temperaturas mínima (Tabela 4A) e máxima (Tabela 4B) de Erechim, com seus respectivos valores de AIC, AICc e BIC. Como sugeriam os correlogramas e periodogramas, os melhores modelos indicados pelos critérios de seleção levam em consideração uma diferença sazonal e não apresentam diferença de ordem 1; os menores valores dos critérios de informação indicam que os modelos que mais se adequaram às duas séries de temperaturas possuem as mesmas ordens, referentes ao modelo SARIMA $(1,0,1)(1,1,1)$.
B.

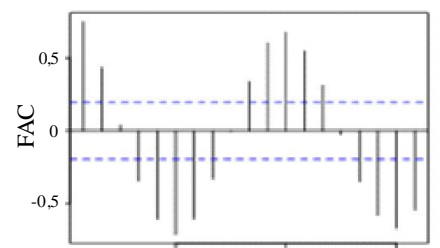

(a) FAC amostral

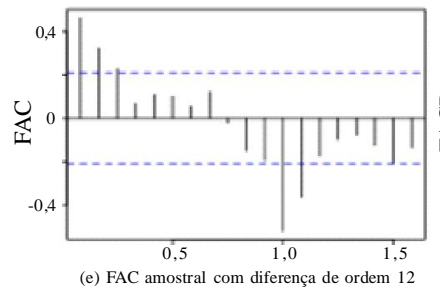

(A) e máxima (B)

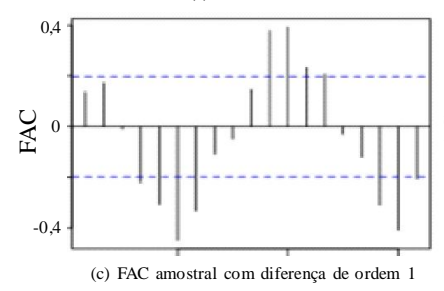

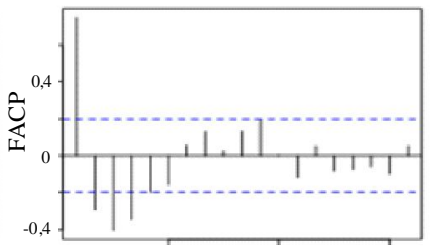

(b) FACP amostral
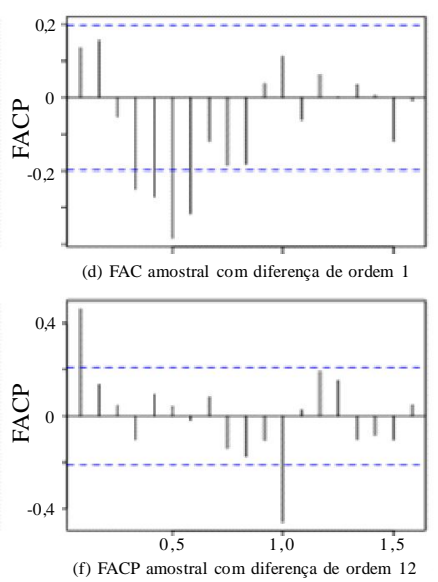

Para avaliar a qualidade de ajuste desses modelos a Figura 7 apresenta a análise de diagnóstico.

A análise de diagnósticos valida o modelo para que as previsões possam ser realizadas. Observa-se que nenhuma autocorrelação residual é significativamente diferente de zero, os resíduos padronizados se encontraram entre -2 e 2 e no teste de Ljung-Box nenhum p-valor ficou abaixo da linha tracejada, indicando que não se rejeita a hipótese nula de erros não autocorrelacionados; conclui-se, então, que este é um modelo adequado para fazer previsões; esta boa qualidade do ajuste também pode ser observada na Figura 8 .

Nos gráficos da Figura 8 observam-se os valores reais na linha cheia e os valores previstos pelo modelo SARIMA $(1,0,1)(1,1,1)$ na linha tracejada, para as séries de temperaturas mínima (Figura 8A) e máxima (Figura 8B). Percebe-se que o modelo é acurado, captando corretamente a variabilidade das séries.

\section{Comparação entre os modelos de alisamento exponencial e da classe ARIMA}

Esta seção apresenta uma comparação entre os modelos Holt-Winters e da classe ARIMA, avaliando qual modelo é mais adequado para previsões das séries de temperaturas mínima e máxima mensais. Para tanto, são utilizadas medidas de qualidade das previsões, conforme Tabela 5, cujos resultados são apresentados para $h=3 \mathrm{e} h=6$, ou seja, ao traçar previsões para os próximos $h$ meses à frente.

De acordo com as medidas de qualidade apresentadas na Tabela 5, observa-se que os modelos SARIMA possuem maior acurácia que os modelos Holt-Winters, superioridade que pode ser verificada pelos menores valores de EQM e MAPE, tanto para temperatura mínima quanto para a temperatura máxima e nos dois horizontes de previsão. Contudo, os modelos Holt- 
Tabela 4. Mel hores modelos SARIM A para as séries de temperaturas mínima (A) e máxima (B) de Erechim, RS

\begin{tabular}{|c|c|c|c|c|c|}
\hline Modelos & Coeficientes & E.P & AIC & AICC & BIC \\
\hline $\begin{array}{c}\text { SARIMA } \\
(2,0,3)(1,1,1)_{12}\end{array}$ & $\begin{array}{l}\text { A. Tem } \\
\phi_{1}:-0,0666 \\
\phi_{2}: 0,6225 \\
\theta_{1}: 0,3667 \\
\theta_{2}:-0,4958 \\
\theta_{3}: 0.1374 \\
\Phi_{1}: 0,1871 \\
\Theta_{2}:-1,000\end{array}$ & $\begin{array}{c}\text { eratura } m \\
0,1818 \\
0,1786 \\
0,2403 \\
0,2565 \\
0,1595 \\
0,1287 \\
0,4160 \\
\end{array}$ & 345,26 & 347,26 & 364,41 \\
\hline $\begin{array}{c}\text { SARIMA } \\
(1,0,2)(1,1,1)_{12}\end{array}$ & $\begin{array}{l}\phi_{1}: 0,8011 \\
\theta_{1}:-0,5725 \\
\theta_{2}: 0,0185 \\
\Phi_{1}: 0,1682 \\
\Theta_{1}:-0,9997\end{array}$ & $\begin{array}{l}0,1578 \\
0,1841 \\
0,1473 \\
0,1267 \\
0,4447 \\
\end{array}$ & 344,35 & 345,48 & 358,72 \\
\hline $\begin{array}{c}\text { SARIMA } \\
(1,0,1)(1,1,1)_{12}\end{array}$ & $\begin{array}{l}\phi_{1}: 0,8106 \\
\theta_{1}:-0,572 \\
\Phi_{1}: 0,1658 \\
\Theta_{1}:-1,0000\end{array}$ & $\begin{array}{l}0,1351 \\
0,1800 \\
0,1253 \\
0,4454 \\
\end{array}$ & 342,37 & 343,17 & 354,34 \\
\hline $\begin{array}{c}\text { SARIMA } \\
(3,0,3)(3,1,1)_{12}\end{array}$ & $\begin{array}{c}\text { B. Temp } \\
\phi_{1}:-1,0728 \\
\phi_{2}: 0,4037 \\
\phi_{3}: 0,6944 \\
\theta_{1}: 1,5489 \\
\theta_{2}: 0,3490 \\
\theta_{3}:-0,3439 \\
\Phi_{1}:-0,1656 \\
\Phi_{2}:-0,0855 \\
\Phi_{3}: 0,1272 \\
\Theta_{1}:-0,9999\end{array}$ & $\begin{array}{c}\text { eratura } m \\
0,1559 \\
0,2613 \\
0,1304 \\
0,2020 \\
0,3883 \\
0,2033 \\
0,1460 \\
0,1500 \\
0,1869 \\
0,5970 \\
\end{array}$ & 367,5 & 371,33 & 393,84 \\
\hline $\begin{array}{c}\text { SARIMA } \\
(1,0,1)(1,1,1)_{12}\end{array}$ & $\begin{array}{l}\phi_{1}: 0,7579 \\
\theta_{1}:-0,3899 \\
\Phi_{1}:-0,1258 \\
\Theta_{1}:-0,9999\end{array}$ & $\begin{array}{l}0,1369 \\
0,1878 \\
0,1137 \\
0,2676 \\
\end{array}$ & 361,25 & 362,05 & 373,23 \\
\hline $\begin{array}{c}\text { SARIMA } \\
(1,0,1)(2,1,1)_{12}\end{array}$ & $\begin{array}{l}\phi_{1}: 0,7189 \\
\theta_{1}:-0,3009 \\
\Phi_{1}:-0,3602 \\
\Phi_{2}:-0,3001 \\
\Theta_{1}:-0,5973\end{array}$ & $\begin{array}{l}0,1425 \\
0,1968 \\
0,2289 \\
0,1938 \\
0,2812\end{array}$ & 361,25 & 362,39 & 375,62 \\
\hline
\end{tabular}

A.

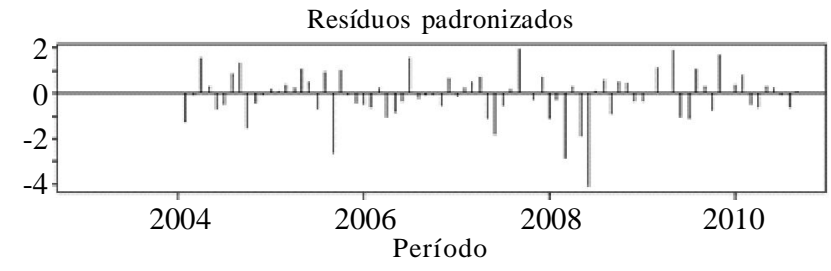

FAC dos resíduos

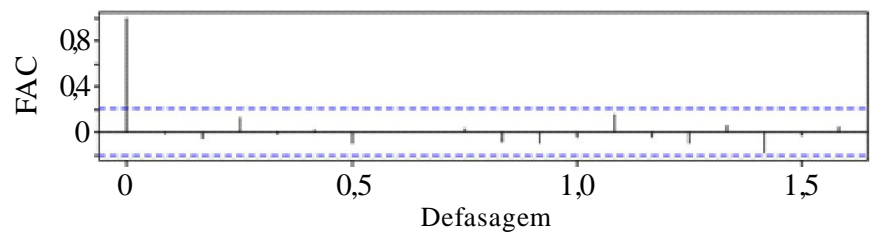

p-valores para o teste Ljung-Box

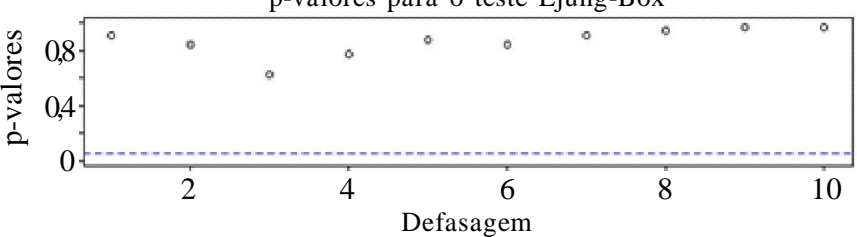

Figura 7. Análise de diagnóstico para o modelo SARIM A $(1,0,1)(1,1,1)$, para as séries de temperaturas mínima (A) e máxima (B) de Erechim, RS
A.

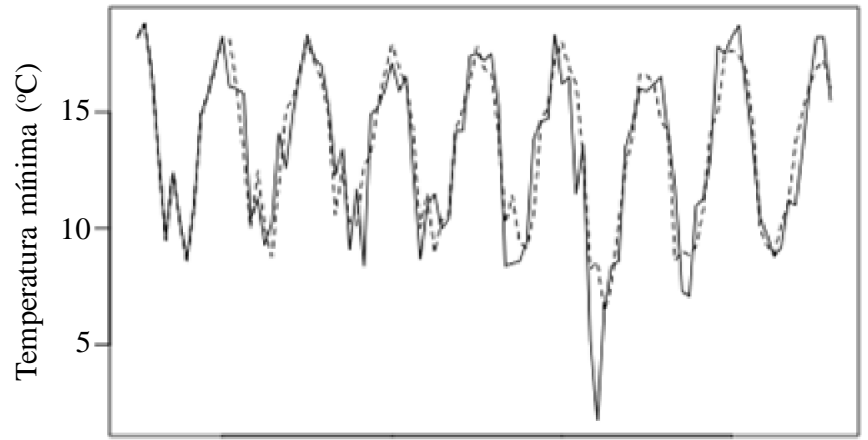

B.

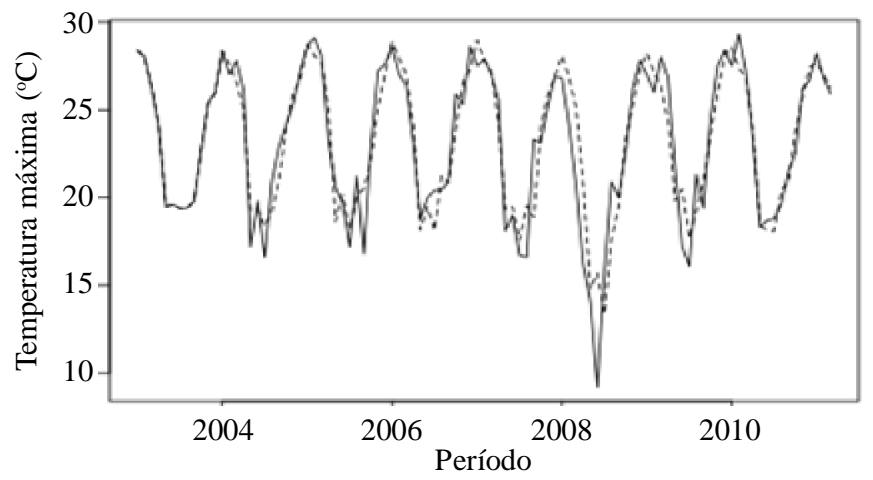

Figura 8. Val ores reais (linha cheia) e valores previstos (linha tracejada) utilizando-se o modelo SARIMA $(1,0,1)$ $(1,1,1)$ para as séries de temperaturas mínima $(A)$ e máxima (B)

Winters também se mostraram adequados para essas previsões, como pode ser visto na Figura 4. Há de se destacar a facilidade de uso dos modelos Holt-Winters, os quais não possuem hipóteses estatísticas restritivas e não requerem análises prévias nem posteriores ao ajuste do modelo. As constantes de alisamento são ajustadas por métodos numéricos simples,

B.
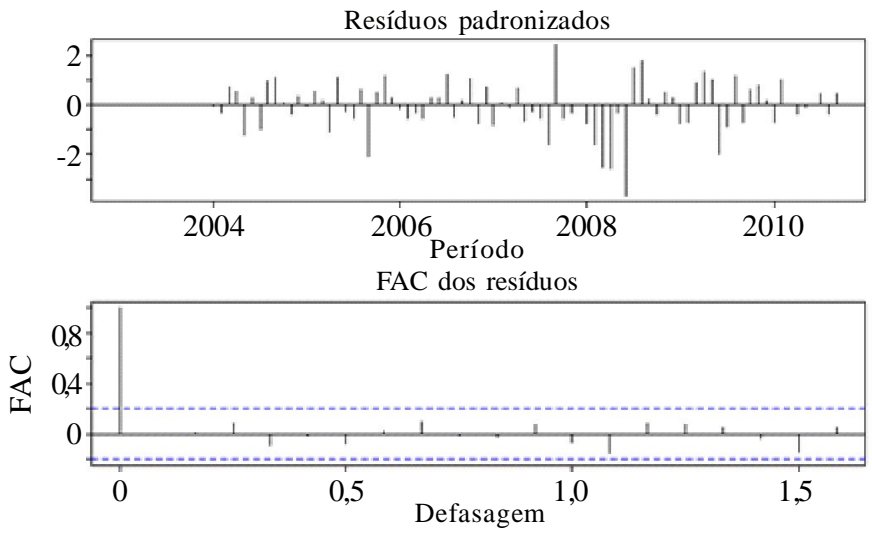

p-valores para o teste Ljung-Box

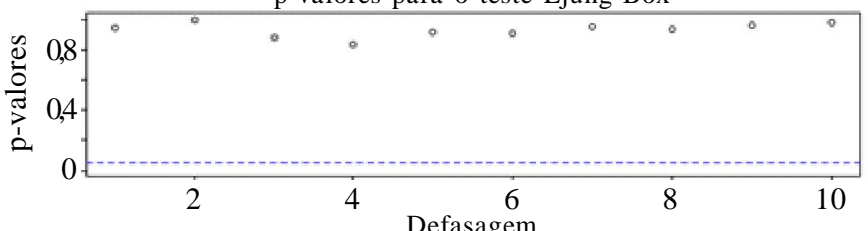


Tabela 5. Medidas de qualidade de previsão para as séries de temperatura mínima e máxima

\begin{tabular}{ccc}
\hline Técnica & EQM & MAPE \\
& $\begin{array}{c}\text { Temperatura mínima } \\
\mathrm{h}=6\end{array}$ \\
Holt-Winters & 2,921 & \\
SARIMA & 2,709 & 0,110 \\
& $\mathrm{~h}=3$ & 0,102 \\
Holt-Winters & 3,971 & \\
SARIMA & 1,412 & 0,104 \\
& Temperatura máxima & \\
Holt-Winters & $\mathrm{h}=6$ & \\
SARIMA & 1,312 & 0,054 \\
& 0,579 & 0,024 \\
Holt-Winters & $\mathrm{h}=3$ & \\
SARIMA & 0,231 & 0,014 \\
\hline
\end{tabular}

EQM - Erro quadrado médio; MAPE - Erro absoluto percentual médio

$$
\text { A. }
$$
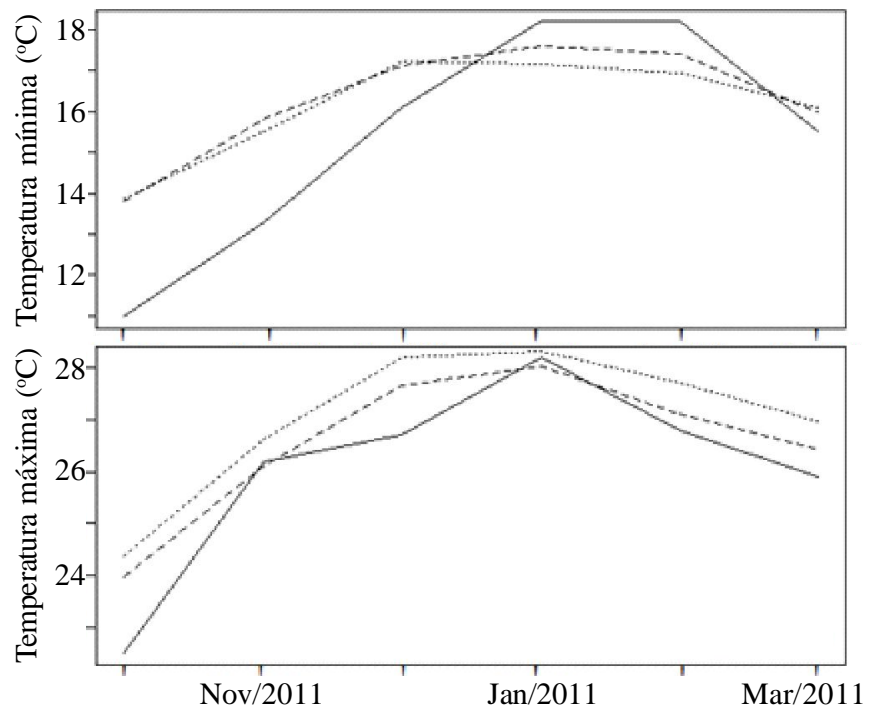

B.
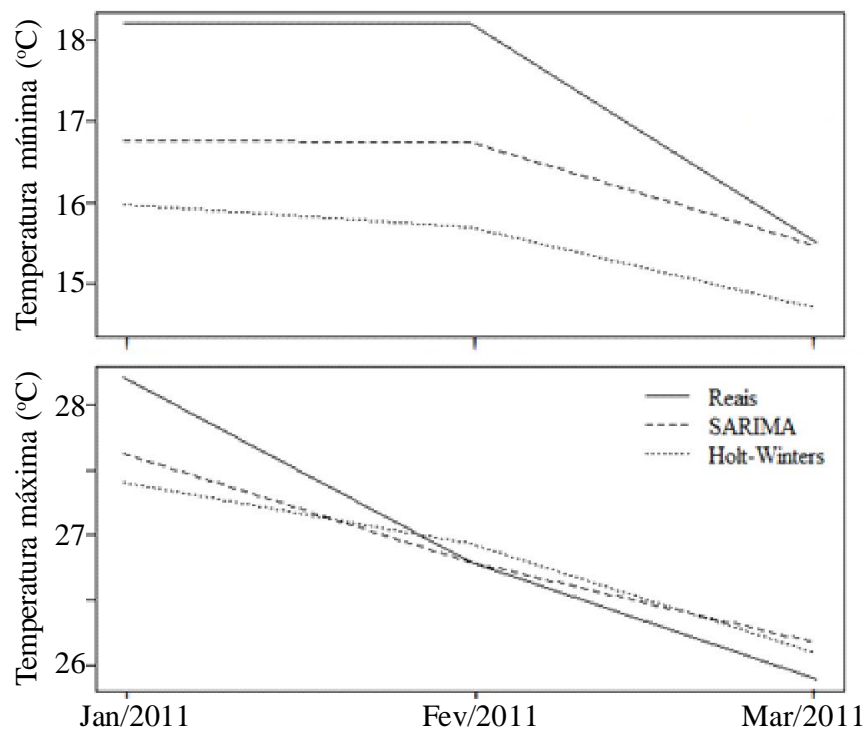

Figura 9. Valores reais (linha cheia), valores previstos pelo modelo SARIMA (linhatracejada) eval oresprevistospel o modelo Holt-W inters (linha pontilhada) para as séries de temperaturas mínima e máxima, com $h=6$ (A) e $h=3$ (B) passosà frente de forma a minimizar o EQM, fazendo com que esses modelos também sejam chamados algoritmos de previsão ou modelos automáticos de previsão.

Na Figura 9 se observam os valores previstos para as duas séries utilizando-se as duas classes de modelos, com previsões seis passos à frente (Figura 9A) e três passos à frente (Figura 9B). Nesta comparação qualitativa também se percebe que os modelos SARIMA levam vantagem sobre os modelos HoltWinters, apresentando valores previstos mais próximos aos valores reais, resultados que corroboram com os resultados quantitativos da Tabela 5 .

As conclusões comparativas sugerem a superioridade do modelo da classe ARIMA; este resultado vai ao encontro dos resultados de Assis et al. (2010), em que o modelo ARIMA foi superior ao modelo de alisamento exponencial na previsão dos preços de feijão e de cacau. Com aplicações em mercado financeiro, Newaz (2008) também verifica a superioridade dos modelos ARIMA frente aos modelos de alisamento exponencial. Por outro lado, os resultados de Kahforoushan et al. (2010) indicam superioridade dos modelos Holt-Winters em alguns casos de previsão do valor agregado de alguns subsetores agrícolas.

Percebe-se que a adequacidade do modelo depende da série em estudo e de suas características. Por meio de gráficos e de medidas de qualidade pôde-se verificar que os modelos da classe ARIMA se mostram mais adequados para modelar as séries temporais investigadas. Com isso, sugere-se o uso dessa classe de modelos como ferramentas quantitativas para o monitoramento, modelagem e previsão da temperatura do ar em Erechim e em outros locais que apresentem características climatológicas semelhantes.

\section{CONCLusões}

1. Verificou-se que as séries de temperatura mínima e máxima mensais de Erechim contêm muitas semelhanças nos seus dados; essas variáveis apresentam sazonalidades e periodicidades bem definidas.

2. Os modelos de séries temporais investigados neste trabalho mostraram-se adequados para a modelagem dos dados climatológicos considerados, apresentando previsões acuradas dentro e fora do intervalo de valores observados.

3. O resultado comparativo foi favorável aos modelos da classe ARIMA; esses modelos foram capazes de captar a variabilidade das séries temporais mensais de temperatura do ar, provendo previsões muito próximas às dos valores reais observados.

\section{AgradeCimentos}

O primeiro autor agradece à Pró-reitoria de Pesquisa e Pósgraduação da UFFS pela concessão da bolsa PIBIC (Edital 003/PROPEPG/2010). 


\section{LITERARURA CITADA}

Akaike, H. A new look at the statistical model identification. IEEETransactions on Automatic Control, v.19, p.716-723, 1973.

Akaike, H. A Bayesian analysis of the minimum AIC procedure. Annals of the Institute of Statistical Mathematics, v.30, p.914, 1978.

Assis, K.; Amran, A.; Remali, Y.; Affendy, H. A Comparison of univariate time series methods for forecasting cocoa bean prices. Trends in Agricultural Economics, v.3, p.207-215, 2010.

Bardin, L.; Pedro Júnior, M. J.; Moraes, J. F. L. Estimativa das temperaturas máximas e mínimas do ar para a região do Circuito das Frutas, SP. Revista Brasileira de Engenharia Agrícola e Ambiental, v.14, p.618-624, 2010.

Bayer, D. M.; Castro, N. M. R.; Bayer, F. M. Modelagem e previsão de vazões médias mensais do Rio Potiribu utilizando modelos de séries temporais. Revista Brasileira de Recursos Hídricos, v.17, p. 229-239, 2012.

Bayer, F. M.; Souza, A. M. Wavelets e modelos tradicionais de previsão: Um estudo comparativo. Revista Brasileira de Biometria. v.28, p.40-61, 2010.

Box, G.; Jenkins, G. M.; Reinsel, G. Time series analysis: forecasting and control. 4.ed. Hoboken, New Jersey: John Wiley \& Sons, 2008. 746p.

Cargnelutti Filho, A.; Maluf, J. R. T.; Matzenauer, R. Coordenadas geográficas na estimativa das temperaturas máxima e média decendiais do ar no Estado do Rio Grande do Sul. Ciência Rural, v.38, p.2448-2456, 2008.

Cargnelutti Filho, A.; Matzenauer, R.; Maluf, J. R. T. Radin , B. Modelo harmônico para a estimativa da temperatura do ar média mensal em diferentes locais do Estado do Rio Grande do Sul. Ciência Rural, v.40, p.1888-1896, 2010.

Cavalcanti, E. P.; Silva, V. de P. R. da; Sousa, F. A. S. Programa computacional para a estimativa da temperatura do ar para a Região Nordeste do Brasil. Revista Brasileira de Engenharia Agrícola e Ambiental, v.10, p.140-147, 2006.

Chatfield, C.; Yar, M. Holt-winters forecasting: some practical issues. Journal of the Royal Statistical Society: Series D, v.37, p.129-140, 1988.

Collischonn, W.; Tucci, C. E. M.; Clarke, R. T.; Dias, P. L. S.; Oliveira, G. S. Previsão sazonal de vazão na bacia do rio Uruguai 2: Previsão climática-hidrológica. Revista Brasileira de Recursos Hídricos, v.10, p.61-72, 2005.

Gemitzi, A.; Stefanopoulos, K. Evaluation of the effects of climate and man intervention on ground waters and their dependent ecosystems using time series analysis. Journal of Hydrology, v.403. p.130-140, 2011.
Hubbard, K. G. Agricultural climatology. Journal of Service Climatology, v.1, p.1-9, 2007.

Hurvich, C. M.; Tsai, C. L. Regression and time series model selection in small samples. Biometrika, v.76, p.297-307, 1989.

Hyndman, R. J.; Kostenko, A. V. Minimum sample size requirements for seasonal forecasting models. Foresight: The International Journal of Applied Forecasting, v.6, p.1215, 2007.

Kahforoushan, E.; Zarif, M.; Mashahir, B. Prediction of added value of agricultural subsection using artificial neural networks: Box-Jenkins and Holt-Winters methods. Journal of Development and Agricultural Economics, v.2, p.115-121, 2010.

Kärner, O. ARIMA representation for daily solar irradiance and surface air temperature time series. Journal of Atmospheric and Solar-Terrestrial Physics, v.71, p.841-847, 2009.

Ljung, G. M.; Box, G. E. P. On a measure of lack of fit in time series models. Biometrika, v.65, p.297-303, 1978.

Medeiros, S. S.; Cecílio, R. A.; Melo Júnior. J. C. F.; Silva Júnior. J. L. C. Estimativa e espacialização das temperaturas do ar mínimas, médias e máximas na região Nordeste do Brasil. Revista Brasileira de Engenharia Agrícola e Ambiental, v.9, p.247-255, 2005.

Morettin, P. A.; Toloi, C. M. C. Análise de séries temporais. Associação Brasileira de Estatística. São Paulo: Edgard Blücher, 2.ed., 2006. 538p.

Newaz, M. K. Comparing the performance of time series models for forecasting exchange rate. BRAC University Journal, v.5, p.55-65, 2008.

PME - Prefeitura Municipal de Erechim. Erechim. <http:// www.pmerechim.rs.gov.br>. 25 Jul. 2012.

R Development Core Team. R: A language and environment for statistical computing. Vienna: R Foundation for Statistical Computing, 2009. 409p.

Salas, J. D. Analysis and modeling of hydrologic time series. In: Maidment, D. R. (ed.). Handbook of hydrology. Fort Collins: McGraw-Hill. 1992. Cap. 19, p.72-149.

Salas, J. D.; Delleur, J. W.; Yevjevich, V.; Lane, W. L. Applied modeling of hydrologic time series. Littleton: Water Resources publications, 1980. 484p.

Silva, M. I. S.; Guimarães, E. C.; Tavares, M. Previsão da temperatura média mensal de Uberlândia, MG, com modelos de séries temporais. Revista Brasileira de Engenharia Agrícola e Ambiental, v.12, p.480-485, 2008.

Soebiyanto, R. P.; Adimi, F.; Kiang, R. K. Modeling and predicting seasonal influenza transmission in warm regions using climatological parameters. PLoS ONE, v.5, p.1-10, 2010. 